\title{
Municipalismo e descentralização administrativa
}

\section{NFLson OMEgna}

A "Revista do Serviço Público" submete, nesta oportunidade, à meditação de seus leitores um ensaio do Deputado Federal Neison Omegna. Desnecessário se torna acentuar a alta categoria dessa contribuição do ex-Ministro do Trabalho, cujos serviços prestados a São Paulo e ao Brasil são do conhecimento geral.

"Municipalismo e Descentralização Administrativa" é um trabalho que resume, com objetividade e clareza, alguns aspectos do Sistema Federativo em função das realidades, interêsses e reivindicações dos Municipios - as Unidades Elementares componentes da Federação.

O panorama das atuais condições que o Pais atravessa é examinado, com rara objetividade e clareza, pelo insigno parlamentar bandeirantes, do ponto-de-vista do funcionamento dos Governos Locais em suas relações com a União e os Estados.

A colaboração intergovernamental, nas três órbitas da Federação, e as exigências de uma crescente descentralização administrativa adquirem novo significado e uma importância cada vez maior no quadro delineado pelo Deputado NeLson Omegna.

Os surtos de industrialização intensiva e as tendências de uma vertiginosa urbanização - na quase totalidade dos Estados - colocam o Govêrno Federal diante de novos problemas que estão exigindo soluções concretas e urgentes. No mesmo sentido atuam fatôres psicosociais de tôda a ordem, dentre os quais se destaca a adesão das Massas à Politicas do Desenvolvimento. O Municipalismo, surge, então, com inesperado vigor e prestigio, não sòmente como uma manifestação da consciência critica das coletividades, mas, ao mesmo tempo, como instrumento, mecanismo ou processo de atuação politica, técnica e cultural a serviço das Prefeituras e Câmaras.

"Municipalismo e Descentralização Administrativa" reflete as novas tendências e perspectivas do Movimento Municipalista no Brasil e demais Paises do Continente Americano 
através da interpretação do Deputado Nelson OMegna, cuja atuação, na Câmara Federal, se tem caracterizado pela eficiência e acendrado patriotismo.

A dinâmica gestão do Representante paulista, à frente do Ministério do Trabalho, credenciou-o, perante a Nação, como Administrador exemplar. O Deputado Nelson OMEGNa conquistou uma posição de singular prestigio, no Pais, em virtude dos atributos de sua personalidade de homem de pensamento e de ação - fato mais importante do que o eventual desempenho das funções de Ministro de Estado. Professor de Ciências Sociais, estudioso dos problemas de Govêrno e Administração, é, ainda, o Deputado Nelson OMegna, um Lider Autêntico do Movimento Municipalista. As Prefeitura e Câmara de todo o Brasil acompanham, com justificada simpatia, as suas incansáveis atividades. Presidente do I Congresso Nacional dos Municipios realizado em 1950, na cidade de Petrópolis, desde então vem se destacando a sua atuação em defesa das reivindicações dos Municipios. Basta relembrat a participação decisiva do ex-Ministro do Trabalho nos posteriores Congressos, nacionais e internacionais, periodicamente levados a efeito pelo Movimento Municipalista. Dentre os Projetos de maior importância que teve oportunidade de apresentar, o de $n^{\circ}$ 4.294-58 obteve excepcional repercussão (Dispõe o mencionado Projeto sôbre o "Manual dos Prefeitos e Vereadores" - publicado pela Revista do Serviço Público - empreendimento de assistência técnica da maior utilidade prática para as Prefeituras e Câmaras, autoridades e administradores municipais, em geral. Por ocasião da VII Reunião do Congresso Interamericano de Municipios, no Rio de Janeiro, em novembro de 1958, foi o Deputado Nelson OMegna escolhido Relator do Tema $n^{\circ}$ II dêsse Conclave internacional ao qual compareceram Delegações de grande número de Paises, inclusive observadores da O. N. U., da U. N. E. S. C. O. da da O. E. A. e diversos outros organismos internacionais. A "Revista do Serviço Público" tem a honra de publicar no presente edição, a Conferência então pronunciada pelo insigne municipalista brasileiro durante uma das sessões plenárias do aludido Congresso: "Municipalismo e Descentralização Administrativa". Não é preciso acentuar que o destacado conferencista recebeu consagradoras homenagens e aplausos de tô das as Delegaçóes, pelo seu magnifico trabalho. Trata-se, efetivamente, de uma contribuição de valor permanente cujo mérito incontestável encontra explicação no fato de ser o Deputado Nelson OMEgNa, simultâneamente, professor, escritor e parlamentar atuante, com admirável experiência - tanto no campo da Politica e da Administração, como no âmbito da Cultura especializada. (Nota da Redação). 
1. MUNiCIPALISMO, MANIFESTAÇÃo DA CONSCIÊNCIA CRÍtiCA DOS POVOS AMERICANOS

Uma das marcas características dos povos latino-americanos em nossos dias, é o aparecimento de uma consciência critica de sua realidade social.

Por inúmeras formas e diversos movimentos, essa consciência coletiva se tem manifestado. Fixemos, porém, um dêles: O Municipalismo.

Em nenhuma época da história continental os municipios foram tão vivamente aproveitados como um grande tema de estudo e análises. Em tôrnodêles, se elaboram campanhas, movimentos de opinião, congressos, centros de estudos, surge a imprensa especializada, criam-se cursos universitários e estruturam-se associações.

Um estado de espírito assim generalizado não surge arbitràriamente. Reflete sempre certas condições objetivas. Coincide o surgimento da consciência crítica que forra o surto municipalista com três processos sociais generalizados nos povos americanos. São êles: processo da industrialização, da urbanização e da adesão das massas politizadas ao processo econômico desenvolvimento nacional.

\section{INDUSTRIALIZAÇÃO COMO OBJETIVO DOS POVOS CONTINENTAIS}

Antigas colônias de plantação e de criação de rebanhos, na sua origem, peruaneceram os países do Continente, por muito tempo, a repetir os moldes da economia colonial. Diversas marcas fixam a fisionomia dêsse ciclo econômico, em que a terra e o homem vivem na dependência dos centros metropolitanos. São êles:

a) O ser uma economia de exploração dentro da qual a formação da riqueza se faz em benefício de valores ou pessoas estranhas ao processo de sua criação;

b) O ser uma economia de complementaridade, isto é, o de existir para suplementar os esquemas de trabalho e produção de outros povos, por isso que se restrinje quase só à produção de matéria prima, que vai alimentar a manufatura de outras nações;

c) O de admitir um como pacto colonial, em que se conforma a aceitar as vedações de produzir qualquer coisa que possa competir com as industrias das nações hegemônicas.

Dentro dessa economia, só participam da vida pública algumas oligarquias, beneficiadas com o regime colonial da produção. E a familia de alguns fundiários, a senhora da administração municipal. Não há povo. As próprias elites se alienam das cogitações da coisa pública. Quando crises internacionais relegaram as produções de exportação à condição de indesejáveis nos mercados estrangeiros e as importações de além mar se tornaram proibitivas, em virtude da guerra, a busca de mercado interno criou um ensejo ao surto industrial. Um indice expressivo do desenvolvimento 
do processo industrial, pelo menos no Brasil, é a mudança da natureza e respécies das importações.

Até recentemente, o pais invertia a maior parte de suas divisas em bens de consumo, para suprir a demanda do mercado interno. Na vintena do século, $80 \%$ do valor da importação do país era de bens de consumo. No último decênio, inverteu-se a situação. A nossa importação, em 1954, era $79 \%$ de bens de produção. Mas o processo do trabalho industrial cria massas interessadas nos planos políticos, engendra uma necessidade de agir, tendo em vista rumos, inculca na psicologia coletiva a preocupação de pensar em têrmos de projeto.

O municipalismo, como vão sentindo os povos americanos, é um empreendimento que visa, sobretudo, a elaboração de novos projetos que mais atendam às populações. E' uma atitude de revisão e de crítica e sobretudo unı comportamento político, irmanado à conduta dos despertados pelos movimentos de industrialização das massas trabalhadoras.

\section{URBANIZAÇÃO, ESTÍMULO AO MUNICIPALISMO}

Outro fato que coincide com o surgimento da ideologia municipalista, é o denso processo de urbanização que vai pela América. No Brasil, confrontando-se os resultados dos censos de 1940 e 1950, observa-se que as cidades em 10 anos acusaram crescimento de $49 \%$, enquanto a população rural acusou um crescimento, em 1950, de só $18 \%$ sôbre o total de 1940 .

O próprio surto industrial vai tangendo para os núcleos urbanos as massas de trabalhadores que vão tentar a promoção econômica dentro das fábricas, e com isso ganham uma fisionomia cultural que as cidades impõem. $U_{m}$ dos traços a aparecer no homem que imigrou é o pendor da política. E' sabido que as condições da vida rural não favorecem o comportamento pròpriamente politico. O velho ruralismo das nossas populações sertanejas que não só fixava o homem à estreita gleba, mas o fazia orgânicamente a ela ligado, como um autêntico elemento da paisagem, e, sobretudo, adstrito a estreito âmbito de inspirações tradicionalistas, de pura imposição ancestral, repetindo hoje o que ontem e anteontem se fizera, e sem possibilidade de alterar amanhã as soluções herdadas dos avós; o velho ruralismo chumba o espirito do homem a uma profunda passividade. Inativo, e inerte, ignora quase sempre a nação e o estado. A urbanização desprende o homem da gleba circunscrita: enche-o de conhecimento técnico dos ofícios; força-o à compreensão dos fenômenos econômicos; dá-lhe a consciência de classe: inculca-lhe direitos; politiza-o, enfim.

Não há dúvida que o aparecimento de uma consciência crítica nos povos do Continente, de que o municipalismo é uma das manifestaçôes, muito se dieve ao processo de urbanização, que por tôda a América se manifesta. 


\section{ADESÃO DAS MASSAS À POLíTICA DO DESENVOLVIMENTO}

Outra alteração que vai coincidindo com a marcha municipalista é a adesão das massas populares às ideologias do desenvolvimento.

Enquanto o povo se ausentava dos processos políticos, entregando-os ao comando das oligarquias, a sua participação no processo econômico era nula. A massa não chegava como ser coletivo a participar conscientemente das atividades dêsse setor. Naturalmente que, como colaboradores individuais, os homens estavam presentes, dando o seu braço ou vendendo sua colaboração. A massa, porém, como consciência coletiva, definindo aspirações gerais, reclamando soluções nacionais, reivindicando benefícios ao povo, programando os roteiros da indústria na demanda do bem de todos, não existia naquele processo, cujos promotores eram apenas as minorias econômicas, que numa atuação de caráter puramente privado, pretendiam tão só alcançar o próprio enriquecimento.

A industrialização e a urbanização das populações americanas promoveram uma elevação da sensibilidade ideológica dos grupos sociais. $\mathrm{O}$ ensejo de participarem ativamente dos pleitos políticos, não como puros rebanhos eleitorais, mas já como elemento conciente, decisivo, reivindicador e, às vêzes, inovador e mesmo rebelde, implantou nas populações um certo pendor empresarial, com que ela vivificou as suas atitudes politicas.

O processo politico empolga o econômico. A massa reclama a sujeição do desenvolvimento material do país às definições programadas dos partidos e dos lideres, impôs a elaboração dos planejamentos, vibrou aos lemas e slogans inspirados na convicção da necessidades de defender os seus minérios. o seu petróleo, as suas fontes de energia, elétrica ou atômica, e proteger as suas indústrias (embora quase tôdas de emprêsas privadas) contra o processo da sua desnacionalização, o que ocorre quando as mesmas se transferem às mãos de alienigenas.

Com isso, vai se sentindo que o processo econômico se torna fato político. E os órgãos governamentais têm de se investir de técnicas, quadros e mentalidades para zelar as fontes de produção e meios de desenvolvimento. Tudo isso é mesmo profundamente revolucionário, pois oferece o processo do desenvolvimento material das nações como fruto de disposições operativas de seus governos, patenteando que a evolução econômica dependa mais da racionalidade das deliberações politicas, que mesmo do arbitrio das minorias empresárias.

Quem acompanha o movimento municipalista na América há de se surpreende com a freqüência dos planejamentos, planificações, programas reclamados pelos congressistas nos plenários. E' muita da luta em que se empenham tantos lideres é só para armar os seus municipios como órgão capaz de fomentar a economia, e bem-estar, o progresso das populações, numa autêntica política de desenvolvimento. 


\section{MUNICÍPIO AUTÔNOMO PARA DESCENTRALIZAÇÃO ADMINISTRATIVA}

Numa civilização trabalhada pela ideologia e imperativos do desenvolvimento, a descentralização administrativa se impõe para dar ao Estado presença nos processos e nas áreas em que a vocação empresarial do povo vai suscitando novos problemas e reclamando prontas providências.

$\mathrm{E}$ aí que o povo, enriquecido com o surgimento de uma consciência coletiva crítica de sua realidade social, acha na velha fórmula de municipio autônomo o órgão essencial à nova fase político-econômica que o país e o Continente vivem, e se empenha nas companhas municipalistas que ai estão florescentes. O objetivo é descentralizar o poder público para fácil solução dos problemas das populações. O caminho que parece mais natural é possuir un município dotado de real autonomia.

A consciência juridica em antiquissimas soluções encontrara essa fórmula já fixada nas cartas forais, nos velhos códigos, onde já surge um govêrno comunal autônomo.

No Brasil, os municipalistas desejosos de ter descentralizado o poder, e dispostos a levar os municipios a planos e realizações, de ordem social, econômica, e até cultural, foram encontrar a autonomia municipal, arma e veículo para uma politica identificada com com as necessidades e as aspirações locais, dentro da própria Carta constitucional. O conceito da autonomia do órgão politico revestia-se desde então das marcas positivas, desde que a lei máxima separava ao órgão do poder local, uma ordem de competência que o faz de certa sorte um como elemento da própria estrutura do regime federativo.

Antecipara-se o tino da nossa liderança politica na Constituinte aos recentes anseios de uma politica de desenvolvimento, situando no Estatuto máximo a melhor fórmula de descentralização do poder, com o município autônomo.

Ao definir os elementos integrantes do Poder, a Carta dá um lugar ao municipio. Marca-lhe o contôrno de sua área de competência e atribuições. Giza-lhe as tarefas. Destina-lhe fontes próprias de receitas.

Aliás, êsse último elementos salienta a consagração do princípio de autonomia. E' sabido pelo depoimento da história dos Estados Unidos, o papel de relêvo que teve a discriminação das rendas na estruturação do Estado Federal. Vale a presença da discriminação, ainda que deficiente nos elementos que reuniu para prover o erário municipal, como um enriquecimento do conceito do município, situado até certo ponto na Carta como uma unidade federativa. Aliás, lembra o ilustre municipalista Doutor Francisco Machado Villa, essa lição ponderando que tal inovação do direito constitucional brasileiro, "é a projeção daquela linha de pensamento de Rui Barbosa, ao afirmar que a autonomia federativa devia infiltrar-se até atingir - municipio, e que tal autonomia não devia parar, quanto à organização municipal, na simples descentralização administrativa, mas ir até à $\mathrm{Fe}$ deração". 
Para a realização de uma política de desenvolvimento, que reclama a descentralização dos órgãos, da administração pública, chegávamos armados da melhor forma: um municipio com a sua autonomia fixada no mais altn e nobre estatuto da Nação, estruturado, no dizer de Pontes de Miranda, como uma entidade intraestatal, rígida como o estado-membro.

\section{AUTONOMIA MUNICIPAL, ALVO DE CONQUISTA}

Tudo que de perfeito e completo a técnica e a consciência juridica dos constituintes legavam aos órgãos da administração local, as condições econômicas do pais, caracterizadas por uma densa centralização financeira e econômica, tornavam grande decepção. E' certo que após a promulgação da Carta de 1946, nestes doze anos já decorridos, houve uma certa evolução política dos municípios, e que as soluções da politica tributária de dar-lhes participação em certas arrecadações da União e dos Estados, representou um relativo adiantamento sôbre as condições anteriores a esta Constituição. Mas. não há dúvida que em face do crescimento das aspirações e anseios populares, dirigidos num sentido de uma politica de desenvolvimento, excitante de planos e de ação, tornou-se realmente frustação e desencanto para a grande parte dos municipios aquelas definições constitucionais.

Até agora uma grande parte dos municipios brasileiros, à conta de fatôres de ordem financeira, econômica, demográfica e técnica, não pode concretizar ạ sta competência legal. Sofre o retardamento em suas conquistas, porque, na marginação em que vive, ou nas distâncias em que se situa, ou na pobreza em que definha, nada lhe tem sobrado da densa centralização financeira, ou do desigual zoneamento econômico que beneficia certas áreas em detrimento de outras.

A luta pela conquista de uma efetiva autonomia e descentralização se deixa inspirar pela convicção de que não basta a simples definição legal, ainda que revestida da autoridade da Constituição, para que o município desfrute a plenitude dos seus títulos e foros. Por isso, os municipalistas mais objetivos e lúcidos, quase todos ansiosos por planificações de largas perspectivas, que criem sobretudo condições econômicas novas à vitalidade municipal, concentram os seus planos de luta nos conselhos e decisöes a que as municipalidades adotem por acôrdos, delegações ou convênios, meios que num primeiro tempo enriqueçam a comunidade, sem o que não lhe será dado o gôzo da própria autonomia.

\section{PLANO, ACÔRDOS E OPERAÇÃO MUNICÍPIO}

Como pensam os municípios conquistar a sua real autonomia?

Os Congressos municipalistas dão uma larga série de soluções a essa indagação. A primeira é a que se propõe a conquistar a reforma da Constituição no capitulo da discriminação das rendas. Não há dúvida que, reforşado o erário municipal, sobretudo com o aumento do seu quinhão dos 
tributos federais, o poder público local cresceria em eficiência, dando melhores serviços às populações a êles jurisdicionadas. Lutam ainda os municípios por uma extensão de atribuições e tarefas, arrebatadas pelos demais podêres, de outros niveis. A grande tareia na campanha de vitalizar os municipios se estriba no programa dos convênios, acôrdos intra-administrativos, pois têm êles um reflexo imediato, dada a possibilidade de funcionarem desda logo, melhorando os niveis de vida das populações. Há uma grande variedade de planos de serviço em aliança entre a União, Estados e Municipios, seja para o reflorestamento das áreas semidesertas, seja para preservação do solo, ou difusão do ensino técnico agrícola, ou mecanização da lavoura, ou alfabetização de adultos, ou instalação das escolas primárias, out obras de saneamento. Essas soluções, aparentemente de âmbito localista $€$ de significado singelo, estão realmente dando condições econômicas às vêzes com absoluto contentamento dos administradores municipais.

Há, porém, um elemento que subjaz à inquietação municipalista, decorrente talvez dessas disposições empresariais, que estão situando o povo no estado do ânimo de influir como fôrças políticas no processo econômico a que nos referimos no início desta exposição. E; por isso que os grandes congressos dos municipios, do Brasil têm se empregado mesmo a fundo na elaboração nuinuciosa, ampla, delongada, cheia de esclarecimentos técnicos, de grandes planos de desenvolvimento econômico. O mais importante dêles, é, de certo, a Operação-Municipio, que já esta hora constitui um projetode-lei do Parlamento brasileiro, e serve de modêlo a diversos projetos em trânsito por diversas Assembléias Legislativas e Câmaras Municipais.

A êste Congresso, o Dr. Araújo Cavalcanti, ilustre municipalista, oferece uma tese, relatando a Operação-Município, que, através de uma política de desenvolvimento econômico-social, visa dar conteúdo ao princípio da autonomia municipal. Para pôr em execução o grande planejamento, esperam os municipios que o govêrno federal, por via orçamentária, ofereça os meios vultosos para se dar às Prefeituras dó país serviços globais, minuciosamente programados, por excelentes órgãos técnicos.

A Operação-Municipio deverá ter uma administração especial, e movimentará fundos destinados ao desenvolvimento de largas áreas dos Estados e comunas que as integram. Sente-se no grande plano uma grande afirmação de fé e de coragem, com que os municípios brasileiros se lançam para conquistar, através de uma prosperidade econômica, aquela mesma autonomia tão perfeitamente delineada no Estatuto constitucional. Daí o aspecto de emprêsa que em certo sentido a Operação assume, e que por isso mesmo entusiasma aos congressos municipalistas do país. E' ela uma mensagem de decidida disposição de fazer da luta pelo desenvolvimento econômico um processo de enobrecimento dos municípios, com a conquista de um novo status de civilização.

$E^{\prime}$ possível que o plano assuste pelo vulto de despesas que implica. Mas na verdade, a receita demandada a Poder Legislativo pelo projetode-lei não nos parece de modo nenhum excessivo. Sem qualquer planejamento de conjunto antes distribuindo estilhaçados recursos e ajudas a tantas instituições, deputados e senadores despendem anualmente quase o que a 
Operação-Municipio calcula para a grande obra de envergadura de valorização de largas áreas do Brasil. O que realmente parece mais dificil seria a organização dos quadros humanos para trabalho em que se reclama tão variada equipe de especialistas. De qualquer forma, o plano se recomenda ao estudo dos ilustres congressistas.

\section{UMA JUSTIÇA DISTRIBUTIVA E A UNIÃO}

E estará realmente certa essa política de valorização de áreas, em que se empenha a Operação-Municipio? De onde virá para a União êsses recursos que ela vai investir nesses setores subdesenvolvidos? Não provirão êles, por ventura, de outros municípios, também cheios de problemas ? Se é verdade que a Operação não discriminará zonas e áreas, mas dispondo-se a realizações em regiões paupérrimas, não virão de outras áreas os elementos financeiros? E atribuindo tais tarefas à União, para que faça derramar às áreas de ralas estruturas econômicas, a cornucópia que vaì encher em outras regiões, não se estará atribuindo ao poder central a justiça distributiva, tirando do remediado ou do rico, para dar ao pobre?

Essas indagações têm a sua resposta, quando se cogita que, numa politica de desenvolvimento, nunca se pode esquecer a necessidade de formação de mercados internos, através do estímulo à elevação dos niveis do poder equisitivo. Não se dissorve às áreas prósperas, quando se levantam os padrões existenciais, das áreas pobres. Mas admitamos se desse à União papel de ministro de uma justiça distributiva, êle é absolutamente justo e certo no processo da politica de desenvolvimento, que constitui realmente uma das molas da campanha municipalista.

O processo do nosso desenvolvimento industrial se faz com alta contribuição de penas, de sacrifícios, de tôda a Nação, e especialmente das áreas subdesenvolvidas.

$E^{\prime}$ sabido que nos paises que emergem do regime colonial, a aglomeração dos capitais de investimento, com que se criam as indústrias, se faz através de processos lentos e difíceis. Logo de saída; o capital nacional tem pela frente, como competidor, os capitais estrangeiros, oriundos de terras onde a mobilização dos investimentos é processo secularmente habitual, e cercados de garantias, nas transações do financiamento e nas organizações das sociedades anônimas. Já estão mobilizados e prontos para aderir aos empreendimentos ao primeiro aceno e se satisfazem, quase sempre, de juros módicos. Para promover os investimentos, o capital nacional luta com a ausência da mentalidade capitalista, enfrenta desconfianças, e paga alto os juros e os prêmios. E' por isso, que tôda indústria iniciante, em países que se desligam da fase colonial, trabalha sempre com o capital espoliativo, o quz agrava diferenciações econômicas, e marca certas áreas, para a condição de metrópole e outras para a de colônia. Parece natural que a União considere o sacrifício que do desenvolvimento de certas áreas impõe a outras, c cuide como um capítulo mesmo dessa politica de desenvolvimento. o assiduo socorro das áreas prejudicadas. 


\section{FORMAÇÃO DE TÉCNICOS}

Há um aspecto curioso nas reivindicações dos municípios para a conquista dos meios para a sua autonomia. E' a variedade de frentes em que êle se situa, a multiplicidade da fatôres que demanda, a pluralidade dos métodos que adota. Já salientamos que, na conquista da condição de órgão descentralizado da administração, os nossos municipios sentiram como problema primordial a grande conquista dos empreendimentos, das oportunidades de uma economia vitalizante. Um elemento se torna imprescindivel na politica dêsse teor, com as suas derivações para os convênios e planejamentos, é a presença do elemento humano.

$\mathrm{E}^{\prime}$ impressionante que tôda a grande movimentação do municipalismo no pais se faz dentro dos quadros da administração, com homens quase sempre desprovidos de cursos de especialidades, como meros autodidatas. O propósito de se aparelhar para o advento dêsses planos que se formulam nos plenários dos congressos, ou nos gabinetes da A.B.M. ou do I.B.A.M., inúmeros administradores municipais vem reclamando da União e dos Estados cursos e oportunidades para o preparo dos técnicos de administração.

O primeiro Estado a se preocupar com o problema foi o de Minas Gerais. Há cêrca de 20 anos criou o primeiro curso para adestrar os funcionários dos seus municipios. Por diversas formas, se pensa dar solução ao problema da deficiência de técnicos para as prefeituras. Primeiro é a formação de cursos, funcionando em triplice modalidade: os cursos universitários, com matérias e especialidades de nível superior, para a graduação dos técnicos para servir na maior esfera administrativa. Há ainda o Curso de aperfeiçoamento, de cadeiras isoladas, para os profissionais liberais que se interessem pela formação de cultura das ciências relacionadas com a administração. E os cursos de treinamento de pessoal, já engajado no funcionalismo, às vêzes funcionando em classes, outras em repartições. Já existe no Brasil duas Escolas de administração pública, com cursos respectivamente de quatro anos, de dois anos e de meses, para aprendizado intensivo. O I.B.A.M. faz funcionar cursos intensivo para treinar secretários e contadores municipais.

Outro método que se tem adotado é o dos cursos de correspondência, pelos quais aliás o funcionalismo do interior tem manifestado o maior interêsse.

Prepara-se ainda o servidor das prefeituras, através de seminários em congregações de número reduzido, para debate de temas determinados e relacionados à melhoria dos serviços e aquisição de adestramento.

Afinal, para satisfazer às instantes solicitações dos administradores municipais, floresce no país já uma apreciável literatura especializada, composta de tratados, cadernos, como os editados pela Escola de Administração Pública ou pelo Instituto Brasileiro de Administração Municipal, e as revistas especializadas, entre as quais se deve destacar a do Departamento das Prefeituras Municipais do Rio Grande do Sul, as notícias municipais do I.B.A.M., a "Revista do Serviço Público" do D.A.S.P., e a "Revista Brasileira dos Municipios", do I.B.G.E. 
Considerando que o vulto da obra de que se encarregam os municípios, e levando em conta que as turmas formadas nas escolas não tiveram tempo ainda de liderar os empreendimentos de natureza administrativa, ou as reivindicações de caráter municipalista, temos que reconhecer que o tato, o tino, a habilidade, a inteligência, o zêlo, o esfôrço, têm armado os homens públicos do município dos melhores propósitos e das soluções mais sábias, para fazer o órgão municipal dar à Nação o vultoso acervo de serviços que vem realizando, com apreciável eficiência.

\section{CONCLUSÔES}

D. expôsto, nos parece certo propôr à recomendação do plenário desta 7 Reunião Interamericana dos Municipios, as seguintes conclusões:

19) As bases da autonomia municipal, definidas na Carta constitucional representam um esfôrço para prestigiar o govêrno da comunidade, em face dos outros podêres governamentais, e armá-lo de condição para melhor atuar como órgão da administração descentralizada.

29) A mera declaração formal não basta para garantir os efeitos da autonomia dos municípios, se a êstes faltam as condições econômicas, financeiras e sociais para a sua atuação efetiva, em benefício das populações.

3) O processo de desenvolvimento material reclama disposições operativas dos podêres públicos, através de planificações, que atendam aos gerais interêsses nacionais.

4\%) A Operação-Municipio representa um estudo sério para a conquista de condições econômicas e sociais dos municípios, dentro de convênio entre os diversos niveis do poder, analisando investimentos para os municípios, promovendo o reerguimento dos padrões de vida das populações e incentivando as fontes da produção. Constitui um profundo estudo de administração planificada, que se impõe à atenção dos municipalistas americanos, preocupados com o subdesenvolvimento das áreas municipais nacionais.

5: A preparação dos técnicos e funcionários municipais pelos órgãos de govêrno federal, estadual, constitui tarefa de real sentido, de utilidade para a melhoria dos serviços públicos, e se recomenda aos senhores congressistas como programa de honesta inspiração municipalista.

\section{BIBLIOGRAFIA}

(1) Medeiros, Océlio de - Problemas fundamentais dos municipios brasileiros.

(2) Almeida. Rônulo de - Os planejamentos econômicos dos municípios.

(3) AzEveDo, Washington - Organização técnica dos municipios.

(4) Araújo Cavalcanti - Desenvolvimento planificado dos municipios do Continente.

(5) Diégues JR. - Agriıpamentos de municipios.

(6) VILlA, Francisco Machado - Justificativa à emenda constitucional da discriminação de rendas.

(7) Costa, Adro:aldo Mesquita da - Fundação dos municipios.

(8) Cadernos do nosso tempo, vol. 4.

* Doc 86, Tema II (VII Reunião do Congresso Interamericano de Municiplos Rio, Novembro de 1958). 


\section{THE MUNICIPALITY AS AN EFFECTIVE UNIT FOR DECENTRALIZED ADMINISTRATION IN ACHIEVING 'THE SEVERAL PURPOSES OF THE STATE}

\section{MUNICIPALISM AS A MANIFESTATION OF CRITICAL CONSCIOUSNESS OF THE AMERICAN PEOPLES}

One of the characteristics of the Latin-American peoples nowadays is their critical consciousnesse of social realities.

This collective consciousnesse has manifested itself in countless forms and various movements. Let us point out but one of them: Municipalism.

In no other time of continental history have municipalities been so thoroughly discussed as a partinent theme for study and analysis. Campaigns are waged for them, movements are raised, congresses are held, centers of studv are created their problems are discussed by the press. In addition, university courses and specialized institutions are being established.

A general state of mind like this does not appear by chance. It also reflects some objective conditions. The rise of this critical consciousness which serves as a basis for the municipal movement coincides with three social processes commen to the American peoples. They are the following: industrialization, urbanization and adherence of politically tterimking masses to the economic procese of national development.

\section{INDUSTRIALIZATION AS ONE OF THE OBJECTIVES OF THE AMERICAN PEOPLES}

As original plantations and cattle raising colonies American nations for a long time used the patterns of colonial economy. Various characteristics bespeak the aspects of this economic cylcle, in which land and man were dependent on metropolitan centers. These characteristics were as follows:

a) An exploiting economy within which wealth was created for the benefit of people or things foreign to ite creation;

b) A supplementary economy, that is to say, an economy which only served for supplementing the work and production of other people. as it was confined almost entirely to the production of raw materials, supplied to the manufactures of other nations;

c) The acceptance of a sort of colonial pact, by which economy was prevented from producing any goods which might compete with industries of master nations.

In this economy, participated only some oligarchies which benefited from the colonial regime of production. Some traditional landowners controlles the municipal administration. The people did not exist. The upper classes themselves ignored public interests. When international crisis caused a negligible demand for export products in foreign markets and overseas 
imports became prohibitive ouwing to war, the interest in the domestic market gave rise to industrial development. An impressive picture of the development of the industrial process, at least in Brazil, is the change which took place in the nature and kind of imports.

Until recently, Brazil used to invest most of its foreign exchange in consumer goods in order to meet the requirements of the domestic market. During the first two decades of this century, 80 por cent of the country's imports consisted of consumer goods. During the last ten years, however, this picture was completely reversed. Our imports of capital goods rose to 79 per cent in 1954. But the process of industrial activities makes people interested in political plans and stimulates action aining at new targets and instils in the collective mind the idea of ever mose projects.

Municipalism, which is now becoming the common cause of American peoples, is a movement which aims principally at the drawing up of new projects which would serve the people's requirements best. It is a revisional and critical attitude and especially a policical bechavior, similar to the conduct of those awakened by the industrial movement of the working masses.

\section{URBANizATION, A STIMULUS to MUNiCipalism}

Another fact which coincides whith the rise of the municipalist ideology is the intensive process of urbanization which is now taking place in America. In Brazil, if a comparison between the census results of 1940 and 1950 is made, it will be not ed that the town population showed a growth of 49 per cent in ten years, whereas the rural population increased only by 18 per cent since 1940 .

The industrial rise itself is impelling the working masses into towns, where they strengthen their economic conditions by working in plants, and acquire also more culture. One of the impeding characteristics of the immigrant from the country is his interest in politics. It is well-known that conditions prevailing in rural life are not favorable to active politics. The traditional ruralism of the hinterland population not only made man settle on a narrow strip of land, but made him attached to it as an integral part of the landscape and, above all, confined him to a restricted field of traditional inspiration, of a mere ancestral nature, repeating today what used to be done formerly and in bygone days with no chance to change in the future the habits interited from his ancestors; in other words, the century old ruralism is a chackle and leads the peasant to a sheer spirit of passivity. Inactive and inert, seldom does he know what a nation or a state is. Urbanization sets man kree from a limited tract of land; affords him a saund knowledgeof arts and craftes; compels him to hunderstand economic facts; gives him a class consciousness; makes him aware of his rights; in short, makes him a politically minded being.

There in no doubt that the rise of critical consciousness of the peoples of the American Continent, one of the signs of which in municipalism is largely due to urbanization which is manifest throughout America. 


\section{ADHERENCE OF THE MASSES TO THE DEVELOPMENT POLICY}

Another change which in now coinciding with the municipalist movement is the adherence of the popular masses to the ideology development.

As long as the people abstained from politics leaving them to the control of oligarchies, their participation in the economic process was nil. The masses, as a collective being, did not participate consciously in these activiities. It is obvious that as individual collaborators men were present in this process, landing or selling their collaboration. The masses, however, did not show collective consciousness, voice general asprovided for an autonomous communal government.

In Brazil, municipalists wishing to decentralize public power and to bring municipalies to carry out plans and accomplishments of a social, economic and cultural order, found in municipal autonomy a weapon and a mean for a policy in line with local needs and aspirations, set forth in the Constitution itself. The autonomy concept of the body politic already showed positive characteristics, since the Constitution gave local authorities a competence which, to tain extent, forms a parte of the very structure of the federal regime.

Our political leaders had wisely provided for the present aspirations of a development policcy, by, including in the Constitution the best way for decentralizing the public power through self-governing municipalisties.

Dn defining the component parts of the Public Power, the Constitution made provisions for municipalisties. It establishes their area of competence, their attributes and their duties and provides them with their own sources of revenue.

As a matter of fact, this last factor stress the principle of autonomy. The prominent role played by revenue distribution in making up the $\mathrm{Fe}$ deral State is well-known in the light of the history of the United States. This fact is noteworthy, although such measure did not fully provide founds for the municipal treasury, because it strengthened the municipal concept, included in the Constitution, as a federal unit. As a matter of fact, this was pointed out by the eminent municipalist. Dr. Francisco Machado Villa, when he stated that such innovation in the Brazilian Constitutional Law "is the continuation of that line of thought which was maintained by Rui Barbosa, when he said that federal autonomy should expand until it would reach the municipality and that such autonomy should not stop, as regards municipal organization, at mere administrative decentralization, but should go as far as the Federation".

For carrying out a development policy, which requires descentralization of public administration organs, we had the best means available: municipalities with their autonomy established by the Constitution, adescribed by the notorious student of Law, Pontes de Miranda, as an interstate body, as rigid as the member-state. 


\section{MUNICIPAL AUTONOMY AS A TARGET}

Everything best that the technical knowledge and the legal consciousness of those who took part in the Constituent Assembly, was given to local administrative bodies, but became a great disappointnent ouwing to our countrk's economic conditions, characterized by great financial and economic centralization. It should be recognized, however, that after the enctiment of the Constitution of 1946, and during the last twelve years, there has been a certain political development om municipalities and further, that the taxation policy entitling municipaliities to receive a part of Federal and State revenue, has accounted for a certain degres of development from conditions prior to this Constitution.

But there is no doubt that in view of strong popular aspirations and feelings, in favor of a develpment policy, leading to plans and action, those constitutional definitions actually became a frustration and discouragement for most municifalities.

Because of factors of a financial, economic, demographic and technical nature, a large part of Brazilian municipalities has up to now not been able $t$ omaterialize theih legal competence. They are hampered by delays owing to their marginal position, or great distances at which they are situated, or their poverty, so that nothing is left over for them from the great financial centralization or the unequal division of economic zones which favors certain areas to the detriment of others.

The struggle for the conquest of effective autonomy and decentralization is inspired by the conviction that it is not enough to have a simple legal definition, even if supported by the autherity of the Constitution, to enable the municipality to enjoy the full use of its rights. Almost all objective and enlightened municipalists therefore advocate planning on a large scale which would above all create new economic conditions for municipal vitality, and they concentrate their plan of campaign on the advice that municipalisties should adopt through agreements, associations and conventions, means which from the beginning would enrich the community wiithout which it will not be able to enjoy ites own autonomy.

\section{PLANS, AGREEMENTS AND THE OPERATION MUNICIPALITY}

How do the municipalities want to conquer their real autonomy?

Municipal Congress made a great number of proposals in answer to that question. The first of them concerns a reform of Constitution in regard to the distribution of revenue. There is no doubt that if the municipal treasury were reinforced, especially through an increase of its share in federal revenue, local government would gain in efficiency and render a better service to the population of its jurisdiction. Municipalities are still fighting for an expansion of their attributes and tasks, taken from then: by the authorities in other government spheres. The great task of the campaign for vitalizing municipalities is to put through a program 
of inter-municipal agreements, for these would have an immediate effect, because they could be put into operation without delay and thus improve the life of the population. There is a great variety of plans for the joint services of the Federal Union, the States and the Municipalities, whether in regard to reforestation of semi-desert areas, or in regard to the conservation of the soil, or spreading of agricultural technical knowledge, or the mechanization of land cultivation, or teaching of adults, or installation of primary schools, or works of sanitation. These tasks which apparently fall into the competence of local government and have a sole signifacence, yield results which are really providing economic conditions that are abso lutely satisfactory for municipal administrators.

There is, however, one factor which causes the municipalist concern and is perhaps a result of these enterprises which are inspiring the people to exercise their influence as a political force in the economic process referred to at the beginning of this exposiition. It is for that reason that the great Brazilian municipal congresses have devoted themselves to an ample and thorough preparation of the great economic development plans, full technical explanations. The most important of these is undoubtedly that called Operation Municpiality, which already has been embodied in a draft of a proposed law (Bill) submitted to Brazilian Parliament, serving as a model for various other Bills discussed by various State Legislative Assemblies and Municipal Chambers.

Dr. Araujo Cavalcanti, a great municipalist, has submitted a paper to the present Congress, in which he deals with the Operation Municipality, which aims at giving a content to municipal autonomy through a policy of economic and social development. In order to put this great planning into practice, the municipalities expect the federal government to provide through the Budget large funds, so that the municipal administrations might possess global services, planned in detail by efficient technical organs.

The Operation Municipality should have a special administration and be able to use funds for the develapment of large areas of the Sstates and Municipalities (Counties) of which the Sstate is composed. The plan is an expression of great faith and courage on the part of Brazilian municipalities, determined to conquer through economic prosperity that authonomy which has been so clearly assured to them in the Constitution. Hence the aspect of enterprise which the Operation to a certain degree acquires and which causes the enthusiasm shown by the Brazilian Congress for it. It shows a determination to make of the struggle of economic development a process for the raising of the prestige of the municipalities by giving then a new status of civilization.

It is possible that the plan might cause anxiety in view of thelarge expenditure which it demands. But as a matter of fact the funds required from the Legislative Power by the said Bill do not seem to us at all excessive. The funds and subsidies devoted to various institutions, deputies and senators annually amount to almost as much as the estimated cost of the Operation Municipality with its great work of valorization of large 
areas of Brazzil. What really seems more difficult is the organization of human resources for this work which requires such a large number of technicians of different kind. In any case the participants of the Congress arz advised to study the project.

\section{DISTRIBUTIVE JUSTICE AND THE UNION}

Is this policy of area valorization aimed at by the Operation Municipality really corretc? Where will the Federal Union find the funds which are to be invested in these underdeveloped sectors? Shall they not perhaps have to come from other municipalities which also have their problems? If it is true that the Operation will not discriminate between zones and areas, yet intends to favor in the first place the poorest regions, does it not imiply that the financial resources will come from the other regions? And by giving such tasks to the Union, expceting it to spend on areas of poor economic structure the surplus carned by other regions, will it not give the central government the power of distributive justice by taking money from the well-to-do or the rich and giving it to the poor?

These questions have their answers, when we consider that in a development policy we must never forget the need of deveToping the domestic market by stimulating th raising of the purchasing power.

It is no disservice to the prosperous areas, if the standard of living of the poor areas is raised. But even admitting that the federal government is given the power to administer distributive justice, this is absolutely right and correct for a policy of development which really constitutes, really, one of the mainsprings of the municipal campaign.

Our industrial decelopment takes place at a great sacrifice of the Nation, especially of the underdeveloped areas.

It is well known that in countries which arise from a colonial regime, the process of accumulation of capital for investments in industries is slow and difficult. At the very beginning domestic capital has to compete with foreign capital from countries, where the mobilization of investments has a centuries old tradition and can count on guarantees, when credit transactions are involved or when joint stock companies are organized. The necessary capital in such cases is available and ready for enterprise at any moment at a reasonable rate of interest. Domestic capital in new countries on the other hand, for the purpose of investments, has to fight with the absence of capitalist mentality and with mistrust, and has to pay a high rate of interest and premiums. That is why all incipient industries in countries arising from a colonial regime have to work with a capital which exploits them which aggravates economic differentiation and marks out certain areas as metropolitan, and others as colonial. It seems natural that the Federal Union should consent to the sacrifice which the development ot certain areas requires from others, and take care of assidous help to underdeveloped areas as part of its policy of development. 


\section{EDUCATION AND TRAINING OF TECHNICIANS}

There is a curious aspect of the claim of the municipalities to gain means for their full autonomy: the variety of factors and methods involved. We have alluded to th efact that, if they want to become decentralized organs of administration, our municipalities should have a strong economy. In this policy, there is an elementa that we cannot dispense with: the human element.

It is strange that all administration of municipalities is entrusted to men with no specialization at all. All Congresses and officers of the Brazilian Association of Municipalities and of the Brazilian Institute of Minicipal Administration, as well as many municipal administrators are asking for courses and opportunities for training administrative technicians.

The first State that tried to solve this problem was Minas Gerais, which twenty years ago founded a course for training its municipal functionaries. There are many methods for resolving this deficiency. First of all, courses are instituted with three aspects: university courses that prepare technicians for the highest administrative sphere; courses for improving those who are interested in administrative sciences; finally, courses for training the personnel already employed in public administration, functioning in school classes, or in public departments. There are already in Brazil two schools of public administration, with courses of four years, two yearsand some months for intensive teaching. The Brazilian Institute of Municipal Administration has an intensive course for training municipal secretaries and accountants. Another method that has been adopted is that of correspondence courses, that have greatly interested officials in the interior.

Municipal funcionaries are also trained in seminaries with a small number of students, where subjects related to the improvement of services and administrative science are discussed.

Finally, in accordance with the needs of municipal authorities, there is a large specialized literature in Brazil, consisting of books, pamphlets, such as those issued by the Public Administration School, the Brazilian Institute of Municipal Administration, and spcecialized reviews; for instance, that of the Department of Municipalities of Rio Grande do Sul, the Municipal Bulletin of the Brazilian Institute of Public Administration, the Review of Public Service of the Administrative Department of Public Service (D.A.S.P.) and the Brazilian Review of Municipalities of the Brazilian Institute of Geography and Statistics (I.B.G.E.).

Considering the importance of the work undertaken by municipalities and the fact that those who graduated in the schools have not yet had an opportunity of directing administrative tasks or municipal claims we must recognise that the tact, skill, zeal, intelligence and capacity of municipal public men have encourage them to work with great efficiency for the benefit of the whole Nation. 


\section{CONCLUSIONS}

In view of the above, we submit to the Plenary Session of the VII Meeting of the Inter-American Congress of Municipalities the following conclusions:

1 - The bases of municipal autonomy, as established in the Constitution, represent an effort to streengthen municipal goverments in relation to other governmental powers and to provide them with such conditions. as to allow them to work as organs of decentralized administration.

2 - A mere formal declaration is not sufficient to assure effective municipal autonomy, if the Municipalities lack the necessary economic, financial and social conditions, to be able to benefit the population in an effective ray.

3 - Material development calls for operative provisions of public authorities by means of plans that taçe into account general national interests.

4 - The Operation-Municipality represents a serious estudy for the conquest of economic and social conditions of municipalities, through agreements among the different levele of government, aiming at investments for municipalities, at the raising of the standard of living of the population and at stimulating production. It is a serious study of planned administration that American municipalists cannot fail to take into account in their struggle against underdevelopment in municipal areas.

5 - The training of municipal technicians and functionaries by the organs of the Union and the States is a really useful task for the improvement of public services. The Congress should study this point because of its sound contnts.

\section{BIBLIOGRAPHY}

(1) Mederros, Océlio de - "Problemas fundamentais dos municipios brasileiros" (Fundamental problems of Brazilian Municipalities).

(2) Almeid (Rômulo de - "Os planejamentos econômicos dos municíçios" (Economic planning of municipalities).

(3) AzEvEdo, Washington - "Organização técnica dos Municipios" (Technical organizatin of municipalities).

(4) ARAújo Cavalcanti - "Desenvolvimento Planificado dos municipios do Continente" (Planned development of municipalities on the Continent).

(5) DIÉGüs JR. - "Agrupamentos de municipios" (Groups of municipalities).

(6) Vilta. Francisco Machado - "Justificativa à emenda constitucional da discriminação de rendas" (Justification for the constitutional emendment of revenue discrimination).

(7) CostA, Adroaldo Mesquita da - "Fundamenta dos municipios" (Foundation of municipalities).

(8) "Cadernos dos nossos tempos", vol. 4 (Pamphlets of our time). 